\title{
INVESTIGACIÓN Y PRÁCTICA EDUCATIVA: LAS TECNOLOGÍAS DE LA INFORMACIÓN Y LA COMUNICACIÓN Y LOS PERFILES DE INNOVACIÓN EN LOS CENTROS ESCOLARES
}

\author{
Laura Rayón Rumayor ${ }^{1}$
}

\begin{abstract}
RESUMEN
En este trabajo presentamos el proceso y una síntesis de los resultados de progreso de un trabajo de investigación iniciado el curso académico 2005/06 en dos centros escolares de educación infantil y primaria en la provincia de Guadalajara. Dicho trabajo se enmarca dentro de la investigación centrada en la escuela ${ }^{2}$, en el que la colaboración entre agentes internos y agentes externos ha conformado un proceso de indagación compartido. En esta primera fase hemos orientado nuestro trabajo a comprender la entidad innovadora de las prácticas de uso de las tecnologías de la información y la comunicación (TIC) en ambos centros. Pretendemos extraer casos y patrones comunes de calidad para el diseño de una guía de innovación que facilite el debate, las iniciativas y la toma de decisiones institucionales en relación con la integración de las tecnologías de la información en el currículo.
\end{abstract}

Palabras-clave: Nuevas tecnologías de la información y la comunicación, currículo, profesorado, innovación, investigación colaborativa, socialización infantil.

\section{INTRODUCCIÓN: NUESTRAS PREMISAS DE PARTIDA}

El estado actual de la investigación en relación con el papel de las TIC en la educación, requiere de diseños y objetivos orientados a generar propuestas innovadoras que definan y expliquen cómo las TIC pueden contribuir a la mejora y el cambio educativo, pero tomando como referencia los contextos de acción reales en los que estos recursos se utilizan y aplican. Con las bases conceptuales contamos, ahora lo que necesitamos es contrastar ese conocimiento contando con las necesidades, posibilidades y realizaciones concretas que se están llevando a cabo en los centros educativos. Se trata de transformar el conocimiento práctico en conocimiento informado a través de un

\footnotetext{
${ }^{1}$ Departamento Didáctica- Universidad de Alcalá. (UAH).

${ }^{2}$ La investigación en el que se inserta el trabajo que aquí se presenta ha sido subvencionada por la Conserjería de Educación de la Junta de Castilla-La Mancha en la convocatoria de "Ayudas para el desarrollo de proyectos de cooperación en materia de innovación e investigación entre el profesorado universitario y no universitario de la Comunidad de Castilla-La Mancha". (Resolución DOCM, 24 de julio 2006, Ref.: E2005-003. Coordinadora Principal: Laura Rayón Rumayor)
} 
proceso en el que el profesorado contraste práctica y teoría por ser éste agente activo que reconstruye y recrea la utilización de estos recursos en la realidad cotidiana de la escuela. Partimos pues de un diseño de investigación y un objeto de estudio que reconoce la importancia de entender la investigación educativa como una unidad entre teoría y práctica, al ser el procedimiento más adecuado para el enriquecimiento tanto de la teoría como de la práctica educativa (Carr y Kemmis, 1986).

Como consecuencia de lo planteado elaboramos una propuesta de investigación marco y contactamos con dos centros escolares de la provincia de Guadalajara con tradición en la innovación educativa, así como con cada uno de los dos Centros de Profesores y Recursos de referencia para cada unos de ellos. Presentamos y debatimos en una reunión inicial el proyecto que fue aprobado por los Consejos Escolares correspondientes y los equipos docentes de los dos Centros de Profesores y Recursos. Tras una segunda reunión de trabajo, conformamos un equipo de investigación mixto, compuesto por dos agentes externos, más un grupo de ocho docentes y dos asesores de formación permanente vinculados a uno de los centros escolares, y otro grupo vinculado al segundo centro escolar- compuesto por dos profesores y otras dos asesoras de formación permanente de referencia.

En una tercera sesión de trabajo en gran grupo se debatió el proyecto de investigación más a fondo, de modo que a partir del problema objeto de estudio debatiéramos y consensuáramos una serie de premisas en torno a las finalidades concretas del proyecto, el sentido de la misma en nuestra formación y los compromisos que de todo ello se derivaban para todos los implicados. Asumimos que el trabajo que iniciábamos tenía que ser un proceso formativo para todos, a través del cual íbamos a conocer cómo funcionaban o podrían funcionar las innovaciones en materia de tecnologías de la información en contextos concretos, atendiendo a los factores que las promueven y consolidan, y a los factores que las dificultan e incluso pueden llegar a fagocitarlas. Desde estas consideraciones, asumimos que los modos de llevar a cabo los proyectos educativos que hagan uso de las tecnologías no iban a depender sólo de la teorización y la investigación pedagógica, que orientadas a la aplicación de una determinada teoría científica dieran lugar a reglas en términos de comportamientos prácticos para el profesorado. Tras las primeras reuniones de trabajo asumimos que "la práctica en educación no espera, normalmente, ni puede esperar a que exista un corpus de conocimiento capaz de regularla, responde a necesidades o son respuestas intuitivas 
a esas necesidades, cuya justificación si se busca y si llega, vendrá más tarde” (Gimeno, 1990:85).

Tres cuestiones se detallaron y desarrollaron en un informe inicial que se elaboró por escrito, quedando como siguen. El primer aspecto importante es el consenso en torno a que la práctica aún siendo un referente básico que orientaría nuestro trabajo, no iba a ser el único, asumiendo que el proceso de investigación se debía de convertir en un proceso de reflexión y de construcción de teoría de la enseñanza de la realidad práctica de los centros escolares. Se prestó especial atención a que el grupo comprendiera que la investigación que iniciábamos podía tener el valor de cambiar y mejorar los modos de aprender y enseñar con estos recursos, pero para ello teníamos que generar un tipo de conocimiento interpretativo y contrastado entre práctica y teoría, y viceversa.

Una segunda cuestión que acordamos fue que las exigencias que la Sociedad de la Información plantea a la escuela, nos exigían el discernimiento de las realidades en la acción para detectar las bondades de la práctica educativa, confirmar la bondad educativa o no de determinados aspectos o dimensiones, y detectar contradicciones y aciertos. Los miembros del equipo asumimos que el trabajo que iniciábamos, parafraseando a Carr y Kemmis (1986:129), no podía confiar en las técnicas y los métodos encaminados a producir teorías; debía adoptar, en su lugar, procedimientos para descubrir teorías en función de las cuales se conducen las prácticas educativa.

Una tercera cuestión consensuada previo debate, y consecuencia de la anterior, fue que la búsqueda de respuestas para una integración curricular de las tecnologías más allá de las clases de informática o de un uso de estos recursos con un sentido instructivo y disciplinar, requería contar con el profesorado, con su manera de percibir y formular los problemas en relación a la integración curricular de estos recursos desde su experiencia cotidiana. Acordamos que en los procedimientos de trabajo los agentes externos no podían desarrollar un "rol de experto". El trabajo que iniciábamos debía romper con la premisa generalizada que se concreta en identificar la escasa formación del profesorado en el manejo de los recursos como causa fundamental de una integración curricular poco significativa o inexistente de estos recursos en los centros escolares. Cuestión particularmente importante dado que el grupo de investigación era diverso en ópticas y experiencias de trabajo sobre esta cuestión: docentes con experiencia en tecnología muy dispar, cargos directivos, coordinadores de TIC y asesores de formación también un grupo profesional muy diverso en la experiencia en el 
ámbito de la formación permanente. Partimos de una evidencia para todo el grupo, si en nuestro sistema educativo la integración curricular de las tecnologías ha estado centrada durante más de veinte años en la alfabetización instrumental y de manejo de las herramientas, la búsqueda de respuestas no puede estar centrada, como solución prioritaria y única, en una formación permanente basada sólo en el conocimiento de las aplicaciones informáticas y en la capacitación de los docentes para el manejo de los aparatos.

\section{Nuestros objetivos, interrogantes y contenidos fundamentales que han guiado la investigación}

Nuestros objetivos concretos se resumieron en los siguientes:

1. Describir y analizar las variables más sobresalientes que caracterizan la integración y uso de las TIC en los centros escolares en términos de innovación, valorando su repercusión en la enseñanza y en el aprendizaje de los alumnos.

1. Realizar una investigación contando con los directamente implicados en la utilización didáctica de las TIC, el profesorado, para indagar sobre la naturaleza de la alfabetización tecnológica y su valor para la construcción de una escuela acorde con las exigencias de la sociedad de la información.

1. Descubrir y experimentar caminos para que las TIC estén presenten de un modo transversal en el currículo, de modo que el concepto de alfabetización tecnológica rompa con la perspectiva instructiva-disciplinar (clases de informática, uso esporádico de las TIC en algunas asignaturas) en torno a la cual se articula el uso de estos recursos.

1. Detectar y comprender qué factores promueven el éxito o el fracaso de la integración de las TIC en los centros, atendiendo a las formas y efectos que adoptan. 
1. Contrastar estas razones con el tipo de mediación que realiza el profesorado entre la utilización didáctica de las TIC y el aprendizaje del alumnado.

1. Obtener una representación de la innovación en relación con los niveles y tipos de integración de las TIC en los centros escolares.

1. Extraer casos y patrones comunes y/o divergentes que sirvan como modelo guía para la integración de las TIC desde planteamientos de mejora en coherencia con los modelos pedagógicos acordes con la sociedad del conocimiento.

Dado que íbamos a trabajar en distintos centros y con profesionales con distintas trayectorias y perspectivas profesionales, y una vez acordado unas premisas de partida que a modo de significados mínimos compartidos nos sirvieran para comenzar a trabajar, centramos nuestra atención en una serie de preguntas de investigación. Con ello pretendíamos concretar y clarificar el objeto de estudio, de modo que aún siendo conscientes que el proyecto en sus contenidos y formas de proceder iba a concretarse y adaptarse a la realidad singular y específica de cada centro escolar, nos sirviera a todos los implicados para "entrar al campo por la misma puerta", si se nos permite la imagen por expresiva. No obstante, las preguntas se formularon de un modo abierto y flexible, como corresponde a los diseños cualitativos de investigación (Goetz y LeCompte, 1988; Delgado y Gutiérrez, 1999; Flick, 2004), de modo que nos permitan guiar la recogida de datos y su interpretación de un modo procesual y holístico, respetando la naturaleza sociohistórica de los escenarios de investigación. Los interrogantes son los siguientes:

$>$ ¿Cuáles son las condiciones que identifican la innovación en un centro escolar? ¿Qué facilita o dificulta los procesos de trabajo en un aula cuando las TIC se ponen al servicio de un proyecto educativo? ¿En los proyectos de "Innovación e Investigación educativa en la acción", y los proyectos europeos que se desarrollan en los centros escolares, a qué procesos y finalidades sirven las TIC? ¿Tienen sentido poner estos recursos al servicio de otros procesos y objetivos? ¿En qué aspectos mejorarían estos proyectos? 
¿Cómo incide la historia formativa de los centros en TIC y la biografía profesional del profesorado en el uso de estos recursos?

$>$ ¿Qué procesos y resultados se logran, más allá del aprendizaje de tipo instrumental (dominio de la herramienta a nivel manejo)? Si se logran otros resultados (actitudinales=ámbito de socialización; procedimentales=análisis de la información, evaluación, contraste, crítica, ...; conceptuales $=$ contenidos significativos,... ¿De qué tipo son, y cómo inciden en el currículo diseñado y el desarrollado?

$>$ ¿Cómo podemos planificar, apoyar y evaluar aprendizajes que tengan que ver con un concepto de alfabetización tecnológica transversal, y estén más relacionados con las posibilidades personales, sociales e intelectuales de los estudiantes, que con el dominio instrumental de la máquina y aplicaciones a ella asociadas?

$>$ ¿Qué barreras tiene el profesorado para poder implicarse en una utilización transversal de las tecnologías?

En relación con los objetivos propuestos e interrogantes formulados, dos contenidos aparecieron como fundamentales en nuestro estudio, el concepto de "Alfabetización Tecnológica" y el concepto de "Innovación". Respecto a ambos conceptos, nos guiarán los siguientes presupuestos:

i. La alfabetización tecnológica cuando queda reducida a la mera utilización instrumental del ordenador y aplicaciones asociadas, es insuficiente para la integración autónoma de los alumnos en la sociedad actual (sociedad de la información y el conocimiento)

ii. En consecuencia, la alfabetización tecnológica cuando se queda en una formación meramente instrumental, se convierte en una realidad educativa y social injusta (brecha digital).

iii. En consecuencia, pasar de una alfabetización tecnológica instructiva a una alfabetización tecnológica formativa, es una cuestión metodológica y pedagógica, pero también social y política (en el sentido de que tiene que ver con aprendizajes imprescindibles que una persona tiene que dominar, su no 
dominio pone en desigualdad a los alumnos frente al trabajo, el ocio, y el acceso a la cultura).

iv. La alfabetización tecnológica de tipo formativo y transversal, debe asociar la experiencia del aprendizaje con TIC con procesos y experiencias en el aula significativas, que partan de la experiencia vivencial de los alumnos, que sin desestimar aprendizajes de conceptos, incida también en una serie de competencias básicas para "sobrevivir en la sociedad de la información".

v. Esta concepción de la alfabetización tecnológica es coherente con una concepción de las TIC como artefactos socioculturales, realidades abiertas que pueden recrearse y mitigar el "determinismo tecnológico" en los modos de pensar e intervenir con estos recursos en la enseñanza.

vi. La contribución de las TIC a la innovación educativa debe partir de un modelo educativo y proyecto curricular que definirán qué y cómo hacer y para qué trabajar con estos recursos, y no a la inversa, que los medios tecnológicos definan estas cuestiones.

vii. En estrecha relación con estas cuestiones, la innovación es un proceso abierto y flexible, que si bien puede definirse de arriba abajo, necesita de una concreción y orientaciones de abajo a arriba como proceso complejo en el que están implicados diversos factores y agentes, quienes otorgan un significado particular en la práctica.

viii.El papel que jueguen las TIC en los procesos de innovación está asociado a los modos en que el profesorado piensa y afronta los cambios, y ello debe contrastarse con las posibilidades que estos recursos ofrecen a lo educativo a través de una investigación sobre la práctica real.

ix. Innovación e investigación educativa se convierten en procesos indisolubles en el que todos los implicados se introducen en proceso de perfeccionamiento y aprendizaje colectivo, en el que el "pensar" y el "hacer" del profesorado debe someterse a revisión de modo que se convierta en una acción informada: praxis.

\section{Formas de proceder y metodología de investigación}


El trabajo realizado hasta el momento se ha desarrollado a través de dos "Estudios de Caso". Las aportaciones que esta metodología nos ha brindado las podemos resumir en los siguientes puntos:

a) El Estudio de Casos se centra en el nivel micro del sistema educativo, particularmente importante en esta primera fase de investigación. Téngase en cuenta que el centro 1, dado las dos experiencias de innovación en TIC que hace tiempo vienen desarrollándose -el Aula de Robótica y la Radio Escolar con los alumnos de $6^{\circ}$ curso-, nos exigen conjugar una recogida de datos atendiendo a perspectivas que contextualizaran los análisis de las experiencias con perspectivas más amplias relacionadas con la organización escolar, la "micropolítica" del centro, y en nuestro caso concreto, el papel que la formación permanente pudiera tener en todo ello. Para el centro 2, el enfoque debía ser el mismo, dado que la innovación, si bien al inicio de la investigación no se identifica claramente con unas propuestas educativas de utilización de las TIC desde parámetros de cambio, sí existen otras propuestas de innovación que necesitamos comprender desde perspectivas más globales.

a) El Estudio de Casos nos permite además poder centrarnos en las situaciones prácticas y en las acciones estratégicas de los participantes, según los diferentes contextos. Así, la estancia prolongada en los centros, ha permitido que los investigadores externos hayamos participado en distintos momentos $y$ circunstancias, recogiendo diferentes informaciones y registrando el curso de las acciones en el aula, en el recreo, en los espacios de exclusiva y en reuniones más formales llevadas a cabo con el grupo de trabajo en cada centro.

a) Y por último, la apertura y flexibilidad que permite esta forma de trabajar nos ha permitido comprender los constructos que los profesores tienen elaborados en relación con la integración de las TIC. Para ello nos hemos movido en dos planos complementarios, que constituyen dos dimensiones básicas de contraste: qué acciones prácticas se llevan a cabo con las TIC y cuáles son los referentes que informan dichas propuestas. Hemos querido desentrañar el "discurso pedagógico" (Gimeno, 1998) en dos planos o niveles: motivos y razones que 
justifican las propuestas de trabajo y su correspondencia concreta con la práctica educativa, atendiendo a los factores que facilitan la innovación y los factores que la dificultan.

La diversidad de técnicas de recogida de datos, observación participante, entrevistas abiertas en los primeros momentos, entrevistas semi-estructuradas posteriormente, y reuniones de contraste y debate, nos ha permitido contrastar lo que hacen y piensan los profesores, generándose un proceso de interpelación reflexiva de la práctica. Hemos cuidado durante el proceso de investigación llevado a cabo controlar las interpretaciones que íbamos haciendo de modo que las inferencias provisionales que se iban construyendo tuvieran sentido para todos los implicados. Reflexionar sobre la práctica haciendo uso de la "filosofía de la educación" -como le gusta decir a una de las investigadoras internas-, ha evitado que el diálogo se convirtiera en una herramienta culpabilizadora por sancionadora en manos de los agentes externos, que como expertos sólo ellos tuvieran algo que decir, puntualizar y aportar.

\section{Los resultados de progreso obtenidos}

A partir de un informe para cada centro, elaboramos un informe multicasos, del cual presentamos una síntesis de los análisis y reflexiones obtenidas.

a) La dimensión social y moral del cambio: el reto de la mirada sociocultural y el sentido organizativo del cambio

Una de las primeras conclusiones que podemos extraer hasta el momento es la concepción del cambio educativo viene alentada por el reconocimiento del valor social de la educación y la función pública de la escuela, que se materializa a través de la organización y se expresa como un convencimiento en la vida de los dos centros a través de múltiples acciones, se haga o no uso de las TIC.

Son centros de puertas abiertas, en donde el tiempo y el espacio escolar se conciben como aprendizaje social. El hacer innovación es para los docentes que la llevan a cabo 
un proceso abierto, que reconocen transgresor porque saben que rompe claramente con el modelo de enseñanza-aprendizaje tradicional, y la disciplinariedad como referente. El alumnado y la comunidad en los dos centros son sus referentes; la voluntad y el deseo, expresado de mil formas y acciones -si se nos permite la imagen por expresiva-, de dotar a los niños y niñas de estrategias intelectuales y actitudes valiosas para incardinarse en la sociedad en la vive es el horizonte.

La preocupación por el mundo que les ha tocado vivir a los alumnos y cómo dar respuesta a estas cuestiones, se concreta en el componente moral de alguna de las propuestas educativas que se llevan a cabo, uno de los rasgos que Fullan (2002) identifica como dimensión fundamental del sentido del cambio en la escuela. Porque como se expresa uno de los investigadores internos: "cuando yo estudiaba un profesor de Pedagogía nos dijo que el maestro tenía que estar a la vanguardia de todo, y yo me lo creí, aunque sea una utopía”.

Y aunque, como es lógico, las propuestas de cambio no están exentas de dificultades y obstáculos, y se asume que la innovación tiene un componente subjetivo personal e irrenunciable, también se viven experiencias de cambio consolidadas y reconocidas públicamente, que tratan de ser el revulsivo al corsé que el currículo normalizado y el libro de texto supone para algunos profesores, que junto con la dirección de ambos centros colaboran abierta y explícitamente en la concreción de la mejora como dinamizadores en la vida de los centros.

"La participación y el equipo" es el lema del primer centro, que se observa claramente en el desarrollo de las propuestas de trabajo en el "Aula de Robótica" y la "Radio Escolar: Onda San Francisco" que llevan a cabo dos profesores, y sobre la que más tarde nos detendremos. "Todos tenemos algo que ofrecer" es el lema del centro 2. $\mathrm{Y}$ en ambos, la dimensión organizativa del cambio se convierte en estrategias y formas de trabajar que se ponen en juego, de modo que haya unas normas y valores que aglutinen y sean la base del proyecto educativo. No se imponen normas de actuación, pero sí se presta atención a sugerir y poner las condiciones para que el profesorado experimente el "valor de educar", parafraseando a Savater. La elaboración de horarios, la planificación de los refuerzos, la participación de los alumnos en juegos tradicionales y alternativos en tiempo de recreo en el centro 1, la participación de los alumnos en la vida del centro en encuentros asamblearios en el centro 2, el plan de animación a la 
lectura en el centro 1, o la semana cultural en el centro 2, son acciones colectivas que se desarrollan para que el profesorado, cada uno desde su compromiso, responsabilidad y experiencia docente, trabajen y colaboren en la escuela, y se hacen esfuerzos para mantener el difícil equilibrio y correlación entre el cambio para la mejora colectiva (equipo docente-alumnos-centro escolar) y el cambio para la mejora individual (profesor-alumno-clase).

El respeto a los profesores y a su singularidad es un proceso que forma parte de la vida del centro. En definitiva, en los dos centros la organización es una organización colectiva que aprende, pero es también una organización "inteligente", en tanto respeta la diversidad de profesores que con biografías profesionales distintas y con concepciones curriculares incluso contrapuestas, no da lugar a "todos a una".

Estos datos evidenciarían otro rasgo del perfil de la innovación de ambos centros, y es tener claro el sentido del cambio. Saben que la innovación tiene que afectar a más de un profesor, la mejora saben también que es para los alumnos $\mathrm{y}$, por tanto, hay que articularla y vivirla no sólo en el aula, sino también el centro, compartirla con quien quiera "saber", explicarla y mantenerla El cambio se concreta en un proceso de aprendizaje y es posible dentro de unos límites, pero en ambos centros se sabe que no puede renunciarse a desarrollar un enfoque de aprendizaje colectivo, para Stoll y Fink (1999), condición importante para la mejora de la escuela.

Estas cuestiones que hemos ido constatando son fundamentales para cualquier centro que en su seno albergue propuestas de innovación, porque -como reconoce Hargreaves (2003:219)- la disponibilidad que una escuela tenga hacia el cambio, entendido este como proceso de aprendizaje continuo, y no como un resultado, depende en buena medida de los profesores. Por ello, valorar a los docentes como personas y sus aportaciones es importante para aumentar su autoestima y generar confianza. En este sentido, una de las conclusiones de trabajo en relación a las posibilidades contextuales que tiene la integración de la TIC, es que cualquier escuela que quiera enseñar en la sociedad del conocimiento, poniendo las tecnologías de la comunicación y la información al servicio de experiencias de aprendizaje para que los estudiantes puedan experimentar la creatividad y la flexibilidad en la realización de tareas, el trabajo en grupo y la autonomía en el aprendizaje, tiene que ser una escuela capaz de desarrollar desde la organización un tipo de profesionalismo abierto, no sancionador o 
culpabilizador para quien no sabe o no está en disposición de hacer de otro modo la educación.

Un tipo de profesionalismo que atienda al trabajo colectivo, poniendo -como los datos evidencian- las condiciones necesarias para que la escuela no convierta la enseñanza en una tarea eminentemente técnica para conseguir unos resultados de aprendizaje aceptables en los exámenes, sino una enseñanza que se sabe y se hace como misión social para que los estudiantes den sentido a su vida y lo que les rodea. Estaríamos ante otro de los rasgos o perfiles de la innovación propios y necesarios para una escuela que quiera responder a los retos de la sociedad de la información. Estos datos son importantes para nuestro objeto de estudio, en tanto perfilan las condiciones básicas que tienen que darse en cualquier escuela para abordar y enfrentarse a los restos que plantea la sociedad de la información.

b) El papel de las tecnologías en las propuestas de trabajo: el coordinador de TIC entre la técnica y la pedagogía

La figura del coordinador de TIC en los centros adquiere una peculiar importancia en relación a las funciones que se le asignen para dinamizar y orientar el uso de estos recursos hacia metas educativas valiosas.

Según los datos recogidos, esta figura hace posible que todo el mundo pase por el aula de informática ${ }^{3}$, pero a costa de un trabajo y esfuerzo que suele tener poca incidencia en la calidad de los aprendizajes del alumnado. El coordinador debiera desempeñar más una mediación pedagógica como dinamizador didáctico, y no una mediación técnica como dinamizador informático. Su rol se concreta en ser informador del software que existe en el mercado, diseñar actividades y tareas en función del producto tecnológico y preparar actividades para sus compañeros, lo que deviene en sobrecarga para el aquel coordinador que quiere cumplir con su trabajo. Como

\footnotetext{
${ }^{3}$ Aula de informática que como dotación inicial la administración educativa de la Junta de Castilla-La Mancha, implantó en los centros escolares. En la actualidad esta aula pervive junto con una dotación de ordenadores portátiles, que bajo un proyecto de conectividad integral en los centros escolares, se empezó a implantar en los centros de educación primaria el curso académico anterior. Destaca del Aula Althia la ubicación de los ordenadores en mesas colectivas de cuatro puestos.
} 
expresaba un miembro del equipo de investigación, el trabajo fundamental que hacemos es un "trabajo de fontanería".

Este planteamiento puede conllevar un peligro, la dependencia de los maestros y maestras con respecto a las tareas del coordinador, que puede devenir en una actitud pasiva ante la integración de las TIC y lo que es más importante, ante lo que hacen sus alumnos frente al ordenador y lo que podrían necesitar aprender.

Otro riesgo que se corre es que algunos docentes vayan asumiendo que las clases de informática o el uso puntual del ordenador no es una práctica sustentadora de los importantes aprendizajes que sus alumnos tienen que llevar a cabo. Esos aprendizajes se llevan a cabo en su aula, con las materias de siempre y las estrategias de toda la vida. Pueden, de un modo implícito oponer una enseñanza basada en medios tradicionales frente a una enseñanza basada en medios electrónicos, lo que a la postre puede llevar a una idealización de la enseñanza y del aprendizaje basado en modelos tradicionales. Se estaría impidiendo un análisis crítico sobre el alcance de la enseñanza basada en los medios de siempre. Cuestión importante si tenemos en cuenta las consecuencias que para la educación obligatoria tiene la "política del libro de texto" (Martínez Bonafé, 2002). De este modo, algunos docentes aunque en su discurso no rechazan abiertamente la incorporación y uso de las tecnologías, en la práctica acaban adoptando una actitud de oposición pasiva ante su integración curricular.

La capacidad reflexiva y crítica que se observa en los dos centros cuando desarrollan los proyectos de innovación, es difícil que se genere en el profesorado que sube al aula de informática, porque, en cierto modo, "los diseñadores son otros". Aunque esta planificación ha supuesto una conquista en los dos centros, pues todos los profesores pasan por el aula de informática, es evidente para el grupo de investigación que en la actualidad hay que superar esta fase. Entre las razones que hemos identificado como debilidades, son las siguientes: los usos de las TIC no son demasiado relevantes, se genera una dependencia técnica maestro-coordinador que absorbe a este último en eso que podemos denominar ya la "fontanería". Además, la utilización de estos recursos es puntual, de manera que se podría estar impidiendo a otros docentes el desarrollo de propuestas con continuidad y significatividad en el aprendizaje de los chicos.

Como aspectos positivos, el aula de informática puede también utilizarse para la realización de tareas para la búsqueda de información, como el profesor del centro 1 
que imparte Lenguaje a los chicos de $6^{\circ}$ curso. Este docente hace uso del aula y de la Internet para apoyar la búsqueda y la organización de los datos necesarios para la emisión radiofónica que en directo que llevan a cabo los chicos todos los jueves, cuando así lo requieren él o sus alumnos. En los dos centros se consigue una meta valiosa como primer estadio o fase por la que tienen que pasar los centros, la modificación de las reglas del juego a las que da lugar un rasgo definitorio del individualismo de la cultura docente dominante: "mi clase, mis alumnos y yo".

Coordinarse, cambiar de espacio y hacer uso de otros recursos didácticos para trabajar con los alumnos, o comenzar a diseñar materiales, como tienen planteado algunos profesores de los dos centros, es otro camino, que si bien no va a garantizar por sí mismo la experimentación curricular con otras metodologías y estrategias didácticas que rompan con una enseñanza transmisora y academicista, si parece condición básica para que algunos docente se replanteen su práctica docente. En síntesis, habría una función más estructural orientada al cambio, que estrictamente didáctica, pero necesaria. El peligro, como hemos analizado con el grupo de investigación, es avanzar en círculos, y mantener quizás una utilización poco relevante.

Parece razonable, a la luz de los datos analizados, que la figura del coordinador en los centros se tendría que concretar no en una tarea tan individual sino reforzarse como una tarea colectiva, de modo que se "rescatara" se "explicitara" el pensamiento y el hacer de los maestros, sometiéndolo a un contraste con nuevas formas de hacer y trabajar en sintonía con los proyectos de innovación que en los dos centros se llevan a cabo desde la perspectiva sociocultural de las TIC. Como posteriormente veremos en el tercer apartado, cuando estos recursos se utilizan con un sentido de transversalidad, el valor educativo del medio se "construye" no en función del programa de turno, si no en clara conexión con el quehacer docente de los dos profesores que las llevan a cabo y con sus creencias sobre qué es la tecnología, qué es la educación obligatoria, o qué necesitan saber sus alumnos más allá de los conocimientos a nivel de usuario de informática.

Las creencias y las acciones son ricas y valiosas en los dos centros escolares cuando los docentes piensan sus prácticas, las explicitan y debaten, independientemente o en relación con las TIC. El sentido pedagógico de Internet, por ejemplo, no es algo intrínseco a la red y sus posibilidades (weblogs, bases de datos, ...), el valor pedagógico 
de uso de esta herramienta, depende de los "saberes" curriculares de los maestros, y en cómo se concreten en la acción eso que saben y con lo que están comprometidos. Y los docentes tienen mucha sabiduría, pero ambas cosas hay que descubrirlas, porque corremos el peligro de obviarlas.

La utilización innovadora de estos recursos precisa de la misma estrategia que utilizan los docentes cuando desarrollan las propuestas de innovación. Habría que anticipar, pensar, prever qué hacemos con las TIC y cómo llevarlo a cabo. Empezar a debatir los fines y las formas de proceder en coherencia con sus aspiraciones y modos de trabajar en el aula, contrastándolo con lo necesario hoy para los chicos. Si no, la identidad profesional del coordinador tal y cómo se concreta en la práctica, deviene en un profesional eminentemente técnico que desestima y obvia el "saber" y el "hacer" pedagógico de sus compañeros, y el suyo propio, porque tiene otras urgencias: mantener el aula de ordenadores, estar al día en programas, ... Esto, como los datos evidencian, individualiza a veces en exceso su rol profesional, y dificulta la emergencia de debates y propuestas que aglutinen a un número significativo de docentes en relación con usos didácticos de tipo transversal con los ordenadores.

Los coordinadores se ven abocados a una autoformación "informática" que les aleja poco a poco del saber pedagógico, interiorizándose en la vida de los centros que la implantación de los recursos tecnológicos es un mero problema tecnológico de presencia de recursos tecnológicos. A lo sumo se considera que la integración curricular de las TIC se ve dificultada o entorpecida porque los coordinadores no están del todo actualizados en programas y aplicaciones, y por tanto, también el profesorado. Y todo ello podría a la larga ir generando una sensación de cierto escepticismo con respecto al papel y valor educativo que pueden tener los recursos tecnológicos en la educación de los alumnos.

A modo de inferencia provisional, pensamos que se podría compaginar la identidad individual del coordinador de TIC con una identidad colectiva, donde las funciones más "técnicas e informáticas" del coordinador fueran subsidiarias de las funciones pedagógico-didácticas de un grupo de trabajo. Hay que fortalecer la figura del coordinador que aglutina el debate y el encuentro profesional con sus compañeros, que dinamiza el centro para pensar qué se puede hacer y cómo traducir eso en actividades con recursos tecnológicos. 
c) Los proyectos de innovación en TIC: la apertura, flexibilidad y el componente transversal

A la luz de los análisis de los datos, el diseño y la puesta en práctica de procesos de enseñanza-aprendizaje con estos recursos desde una perspectiva de cambio, exige pensar el papel de las TIC y concretar los proyectos de trabajo desde un discurso y un hacer eminentemente curricular (procedimientos de trabajo, capacidades a desarrollar, conocimientos a adquirir). En estos casos, y esto es importante, el "viaje" no es de la tecnología a la práctica educativa, sino de su práctica educativa a la tecnología. Es decir, las acciones prácticas a las que dan lugar el "Aula de Robótica" y la "Radio Escolar: Onda San Francisco", son acciones educativas potentes que se han concebido sobre lo que pueden hacer con las TIC desde parámetros pedagógicos, de modo que la utilización de los recursos tecnológicos no se convierte en un fin en sí mismo, sino en un medio facilitador de ambientes de aprendizaje relevantes desde un punto de vista educativo.

Resulta significativo como el profesor que ha diseñado las propuestas, parte de una concepción de la tecnología como una herramienta "muy poderosa, las más poderosa que ha construído el hombre" -como ha expresado en múltiples ocasiones-. Sabe, y es consecuente en su práctica, que las TIC son artefactos socioculturales que nos ofrecen la posibilidad de experimentar potentes procesos cognitivos y de comunicación. Se evidencia que en su práctica docente la tecnología genera unos procesos de pensamiento y actuación ricos y estimuladores. Así, los chicos, al tiempo que trabajan procedimientos de tipo instrumental - los estudiantes aprenden a programar en LOGO en una primera fase, para poner en funcionamiento una serie de artefactos que construyen con material de desecho-, trabajan también procesos intelectuales y actitudinales. En este sentido el aprendizaje social se revela como una dimensión fundamental, en el que el trabajo en grupo y las responsabilidades que de ello se derivan son para los estudiantes su ambiente "natural" de trabajo en el aula.

El distanciamiento con la tecnología y sus aplicaciones que se observa en la actitud profesional de este profesor le permite no sucumbir a usos superficiales y pocos relevantes, de modo que estos recursos y los programas de turno se conviertan en 
herramientas de control pedagógico y social de sus prácticas y del aprendizaje de los chicos. La biografía profesional de este docente le ha facultado para preguntarse por la utilidad de las tecnologías desde intereses pedagógicos, y no meramente instrumentales o técnicos. Como bien asume, durante los primeros años de implantación del proyecto Atenea se deslumbró con estos recursos, y asignó a las tecnologías ese poder de cambiar la educación por el mero hecho de utilizarlas. Reconoce que sucumbió a ese discurso mítico y totalizador que acompañó los primeros proyectos de integración de las TIC en el sistema educativo español.

Sin embargo, ahora insiste en la necesidad que cualquier docente tiene de interrogarse por qué y para qué hacer uso de estos recursos en la educación obligatoria, insiste en que no hay que dejarse llevar, y saber en qué utilizar estos recursos teniendo claro los potentes procesos intelectuales y de comunicación que permiten. Ese hacer algo útil con la tecnología le llevan a realizar un viaje del currículo a la tecnología y no de la tecnología la currículo, viaje en el que la concepción de la educación obligatoria como un proceso no propedéutico, con un valor en sí misma, en el que destaca abierta y claramente el componente social de esta etapa, es una nota dominante. Entiende que la educación debe ir encaminada a ofrecer los instrumentos básicos en el desarrollo de hábitos para aprender a aprender y siente la necesidad como docente de fomentar la autonomía no sólo para afrontar el estudio, sino también para conformar un sujeto socialmente más independiente. Los dos profesores tienen claro que la educación obligatoria no es un tramo de carácter propedéutico, y desde ahí dotan de sentido pedagógico a la tecnología.

Otro rasgo de las creencias pedagógicas de este docente, es que asume abiertamente que la enseñanza no puede centrarse únicamente en la enseñanza de contenidos conceptuales, como evidencia su práctica, y confirma en entrevista con uno de los investigadores externos, entiende que los planteamientos actuales en relación a cómo se concretan los procesos de enseñanza-aprendizaje tienen que cambiar. Si no es evidente que las tecnologías no servirán a procesos educativos valiosos.

Es curioso como el sentido que otorgan al uso de las TIC está en clara relación con las finalidades y el valor que dan a la tarea de educar más allá o más acá de las tecnologías. Y con ellas y con el sentido que otorgan a esas finalidades, consiguen trabajar "procesos" educativos en el que ponen en juego competencias-capacidades coherentes con la sociedad de la información. 
Así, no es de extrañar que este profesor altere de un modo natural la gramática disciplinar que subyace en los planteamientos centralistas que conlleva toda Aula de Informática. Como se ha evidenciado, nos encontramos que la propuesta de innovación del Aula de Robótica se desarrolla de modo que los ordenadores "se mueven", lo que permite trabajar con ellos en dos aulas, la propia aula de $6^{\circ}$ y el aula taller. De este modo se consigue que estos recursos medien en actividades de enseñanza y aprendizaje flexibles y creativas, consiguiéndose un perfil claro de innovación: la tecnología se pone al servicio del profesor y sus alumnos desde la recreación del espacio, alterando las interacciones entre el sujeto y la máquina.

Esto que puede parecer ingenuo, o al menos, poco relevante, tiene una significación pedagógica potentísima. Los datos recogidos evidencian que las actividades que realizan los alumnos en el Aula de Robótica, se crean las condiciones necesarias para que los niños puedan construir sus propias propuestas de trabajo, ser protagonistas de su aprendizaje, o dicho de otro modo, aprender a aprender desde el cambio creativo. Un rasgo que Martín Carnoy y Manuel Castells (Citados por Hargreaves, 2003:34), señalan como imprescindible en tanto, "el trabajo de hombres y mujeres está siendo transformado por las nuevas tecnologías, pero las instituciones sociales necesarias para apoyar este cambio se están quedando atrás”.

En una primera fase, el primer trimestre, los ordenadores se utilizan en el aula de referencia de $6^{\circ}$ curso, y su uso es individualizado. Es cuando adquieren los procedimientos de programación en LOGO. En la segunda fase, una fase de diseño del artefacto, se van al aula taller, que cuenta con todo el material y herramientas para la construcción de los artefactos con materiales de desecho. Y allí se observa claramente cómo se potencia la libertad de movimientos y la diversidad de contenidos que los chicos y chicas trabajan, que no son principalmente conceptuales, sino también -y ahí reside el uso creativo del ordenador- también de tipo procedimental y actitudinal. Los alumnos experimentan el trabajo en equipo, y el esfuerzo que ello supone, pues ellos mismos reconocen lo difícil que a veces es ponerse de acuerdo con respecto a qué construir, cómo hacerlo, qué material es el más adecuado para subsanar un fallo. De este modo, la puesta en común sobre cómo va el trabajo, identificar "los fallos" para subsanarlos, pues son ellos los que crean, son ellos los que se confunden, son ellos los que buscan alternativas para solucionar el problema. Se da un verdadero aprendizaje de 
estrategias cognitivas en relación complementaria con el aprendizaje social. Toman decisiones sobre qué quieren hacer, para qué y por qué lo van a hacer, son ellos mismo los que lo planifican, lo desarrollan y lo ponen en marcha. Y todo ello tiene que quedar registrado, sesión tras sesión, por escrito.

Se observa la tranquilidad, naturalidad y espontaneidad con que los chicos se mueven. Hay aprendizaje porque hay una actividad mediada por la tecnología rica, que a veces es dura para ellos, pero gratificante, como demuestra la ilusión y satisfacción que se observa cuando explican lo que están realizando, o ya han finalizado. Los chicos son protagonistas de una propuesta o proyecto en la que los procesos de trabajo son crear-construir-y "obtener un resultado", como recalca el profesor responsable de la propuesta. Tantean, experimentan de un "modo inteligente", en tanto el profesor media en la actividad, atento no sólo a los resultados, sino a los procedimientos que quiere que aprendan, uno de los principios fundamentales de la pedagogía de Freinet ${ }^{4}$

Estas datos evidenciarían lo que Hargreaves en su trabajo "enseñar en la sociedad de la información" denomina creatividad social, necesaria para el cambio en la escuela. Para este autor los problemas que las escuelas tienen planteado en la actualidad, no son tanto la ingenuidad técnica, sino la ingenuidad organizativa y social. Este autor hace un análisis comparativo entre los centros de primaria y secundaria de Inglaterra en relación a la organización de los ordenadores en los centros, señalando que las escuelas de primaria han mostrado una gran creatividad al incluir los ordenadores en las clases normalizadas, de modo que se permiten procesos de enseñanza y aprendizaje flexibles. Sin embargo, los centros de secundaria al instalar los ordenadores en laboratorios separados, dejan intacta la gramática tradicional de escolarización con su sistema de una asignatura, un profesor, una clase.

\section{Para seguir pensando}

Los centros se mueven, hay condiciones y ganas para seguir experimentando el cambio, y hay saber y buen hacer. Y en este contexto, quizás la formación del profesorado tenga que suavizar y compaginar la formación para una mera alfabetización 
técnica de programas y aplicaciones, con una formación como espacio para la experimentación curricular y la mejora de la escuela, concebida así hace ya tiempo, y que sigue teniendo plena vigencia. Los datos analizados hasta el momento, aconsejan que al afrontar el cambio posible y necesario que puede llevarse a cabo con las TIC, no se haga de un modo reduccionista. Porque, como plantea Fullan (2002:103), cuando las razones que justifican un cambio no están claras, y el profesorado no ve la necesidad del cambio planteado, éste termina siendo más superficial y aparente, que real. El segundo paso, sería clarificar a qué fines educativos podría contribuir su utilización en la educación obligatoria. Téngase en cuenta, que para el profesorado integrar y hacer uso de las TIC desde lo que puede hacer los programas y aplicaciones, puede ser un objetivo claro, pero no lo suficientemente significativo como para que los docentes se pongan en situación de re-pensar y re-crear sus prácticas educativas.

Por todo lo expuesto hasta el momento la formación del profesorado debiera de trabajar, como una primera fase o etapa, contenidos que permitan a los profesores desentrañar en clave pedagógica la relación interdisciplinar entre la cultura tecnológica y la cultura escolar. Las Tic en la formación del profesorado podrían convertirse en la posibilidad necesaria para que el docente:

1. Transformara en clave de aprendizajes los conocimientos que permiten a cualquier persona situarse en una sociedad interconectada, que caracterizada por flujos de información constantes y cambios socioculturales potentes ${ }^{5}$, requieren de destrezas, capacidades y actitudes propias de una educación transversal. ¿Por qué hay que integrar las tecnologías en los proyectos educativos? ¿Necesitan los alumnos saber algo nuevo? ¿qué razones, realidades justifican esos saberes nuevos? y ¿en qué lugar dejamos los viejos saberes de la escuela de los que no podríamos prescindir? ¿Cómo concretamos en metodologías y estrategias un uso de las TIC acordes con lo que necesitan saber nuestros alumnos? En esa búsqueda de razones y argumentos que justifiquen la utilización de las TIC más

\footnotetext{
4

5 Piense el lector, por ejemplo, la necesaria reflexión que los profesores debemos de realizar sobre los cambios en los nuevos escenarios laborales, pues en su dinámica y consecuencias para los futuros trabajadores encontraríamos parte del sentido y la urgencia de facilitar a las nuevas generaciones procedimientos de trabajo intelectual para el aprendizaje autónomo.
} 
allá de los argumentos impuestos por la moda y el mercado, es la tarea en la que el grupo estamos enfrascados.

2. Consecuentemente, también sería una oportunidad para que el docente analizara y clarificara qué grandes metas educativas podrían ser satisfechas y trabajadas en las aulas. Los profesores somos quienes traducimos y llenamos de significado los fines de la educación desde nuestras motivaciones, intereses y conocimiento profesional. Se trataría de subjetivizar lo "nuevo" en relación con las "viejas" metas, que sintieran que la utilización de las Tic es algo valioso que merece ser trabajado en las aulas porque "leen" con claridad los fines educativos, y los traducen en procesos claros y realistas. Esto no significa que la integración de las tecnologías tenga que ser traducida en pequeños fines, porque como nos recuerda Fullan (ibid:105) los cambios simples e insignificantes pueden ser muy claros, pero poco relevantes en el momento de implementarlos y concretarlos en prácticas sólidas y de continuidad en los centros educativos.

Se trataría en definitiva que los docentes buscaran razones para la utilización de las tecnologías y sus aplicaciones en coherencia con las necesidades sociales y educativas que tiene planteadas la escuela. Una búsqueda debatida y consensuada para que las hicieran suyas desde sus compromisos e intereses. Hemos constatado que los docentes tenemos unas creencias más o menos explícitas sobre las razones y fines que satisface el uso de las tecnologías en la educación obligatoria, y son estos supuestos los que se tienen que someter a revisión. Entendemos que mientras estos contenidos no se rescaten en la formación del profesorado, y se anteponga su tratamiento a favor de la alfabetización meramente instrumental de los aparatos y aplicaciones, nos estaremos cerrando a la búsqueda y el encuentro de alternativas valiosas para una intervención de cambio positivo en la práctica educativa.

\author{
RESEARCH AND EDUCATIVE PRACTICE: INFORMATION AND \\ COMMUNICATION TECHNOLOGY AND THE INNOVATION IN SCHOOLS
}

SUMMARY 
In this document we present the process and a synthesis of the progress results of a research work initiated during the school year 2005/06 in two Nursey and Primary State Schools in Guadalajara Region. Such work is framed within a research centred in the school, in which collaboration between internal and external agents has established a shared research process. On this first stage we have orientated our work towards an understanding of the innovative practice of the use of information and communication technology in both schools. We intend to create common models in order to design an innovation guide that enable debates, initiatives and institutional decisions in relation to the fully integration of information and communication technology in the curriculum.

Key Words: New Information and Communication Technology, Curriculum, Teachers (Staff), Innovation, Collaborative Research, Children`s Socialization

\section{BIBLIOGRAFÍA}

CARR Y KEMMIS, (1986). Teoría crítica de la enseñanza. Barcelona: Martínez Roca.

DELGADO Y GUTIÉRREZ (1999). Metodología y técnicas de investigación en las Ciencias Sociales. Barcelona: Octaedro

FLICK (2004). Introducción a la investigación cualitativa. Madrid:Morata.

FULLAN, M. (2002). EI sentido del cambio. Barcelona:Octaedro

GIMENO SACRISTÁN, J. (1990). “Conocimiento e investigación en la práctica educativa". Cuadernos de Pedagogía, 180, 80-86.

GIMENO SACRISTÁN, J. (2001). Educar y convivir en la cultura global. Madrid: Morata.

GOETZ Y LECOMPTE (1986). Etnografía y diseño cualitativo en investigación educativa. Madrid: Morata

HARGREAVES, A. (2003). Enseñar en la sociedad del conocimiento. La educación en la era de la inventiva. Barcelona:Octaedro. 
MARTÍNEZ BONAFÉ, J. (2002). Trabajar en la escuela. Profesorado y reformas en el umbral del siglo XXI. Madrid:Miño y Dávila.

PEYRONE, H. (2001). Célestin Freinet: Pedagogía y emancipación. México:Siglo XXI

RAYÓN RUMAYOR, L. (2003). "Las tecnologías de la información y la comunicación: una perspectiva socio-cultural en el currículum”. Revista Ciencias de la Educación, $n^{\circ} 195$ (Disponible versión en inglés: "Theory and Practice in Educational Techonology: Reflections and Contributions from a sociocultural perspective of the currículo". Revista Virtual de Tecnología Educativa. www. dewey/uab.es/pmarques/EVTE)

STOLL, L. Y FINK, D (1999). Para cambiar nuestras escuelas. Barcelona:Octaedro 